\title{
An efficient and clean synthesis of thiophenyl thiazole depended novel triazolo[4,3-a]quinoxaline derivatives
}

\author{
Haresh G. Kathrotiya, Sagar P. Gami, Yogesh T. Naliapara* \\ Department of Chemistry, Saurashtra University, Rajkot-360005, Gujarat, India \\ *E-mail address: naliaparachem@yahoo.co.in
}

Keywords: Thiophenyl; Thiazole; [1,2,4]triazolo[4,3-a]quinoxaline; lodobenzene diacetate

\begin{abstract}
A simple and efficient approach for the synthesis of thiophenyl thiazole based triazolo[4,3-a]quinoxaline derivatives is described. In this methodology, 3-hydrazinyl- $N-(4-$ (thiophen-2-yl)thiazol-2-yl)quinoxalin-2-amine derivatives treated with various aromatic aldehyde to form Schiff base which on treatment with iodobenzene diacetate in dichloromethane at room temperature to furnish title compounds. The synthesized compounds were characterized by ${ }^{1} \mathrm{H}$ NMR, ${ }^{13}$ C NMR, FT-IR, elemental analysis, and mass spectral data.
\end{abstract}

\section{INTRODUCTION}

1,2,4-triazoles are very interesting targets for medicinal and pharmaceutical applications. The recent literatures are enriched with progressive findings about the synthesis and pharmacological action of fused heterocyclic systems. The structural diversity and biological importance of nitrogen containing heterocyclic systems have made them attractive synthetic targets over many years and they are found in various natural products [1]. Quinoxalines are an important class of nitrogen containing heterocycles with a variety of biological activities. In particular quinoxaline scaffolds were found as a core unit in a number of biologically active compounds. These include anticancer [2, 3], antibacterial [4], antiviral [5], anti-inflammatory [6], anti HIV [7, 8] and antihelmintic activities [9]. Quinoxaline derivatives are also used in the development of novel organic dyes and organic semiconductors. Triazolo [4,3-a]quinoxaline [10] have been reported to possess antiviral, and antimicrobial activities. Many other triazolo quinoxaline derivatives have been reported [11-13] to possess other types of biological properties. Thus, triazolo quinoxaline derivatives continue to attract much attention as these molecules are of potential biological interest.

For synthesis purpose, triazolo quinoxalines can be achieved in several ways. Reaction of 1(2-chloroquinoxalin-3-yl)hydrazine and triethyl orthoformate gave triazolo quinoxaline [14]. Reactions of benzyl bromide or benzyl cinnamate with $N$-(benzotriazol-1-ylmethyl)arylimidoyl chlorides in the presence of $t$-BuOK occur with opening of the benzotriazole ring affording 1,2,4triazolo[1,5-a] quinoxalines [15]. Reaction of $N^{\prime}$-(1,2-dihydro-2-oxoquinoxalin-3-yl)benzohydrazide and HMPA formed triazolo quinoxaline [16]. By heating 2-hydrazino 3-phenylquinoxaline with phenyl-1,3-butanedione produced triazolo quinoxaline [17]. $N$-(2-chloro-4a,8a-dihydroquinoline3yl-methyl)- $N$-3-chloro-quinoxalin-2yl) hydrazones cyclised by iodobenzene diacetate under microwave irradiation technique to furnish the respective quinolinyl-1, 2, 4-s-triazolo [4,3a]quinoxalines [18]. Here we report an efficient and clean synthesis of thiophene thiazole depended novel triazolo quinoxaline derivatives by cyclisation reaction. The constitutions of all the products were confirmed using ${ }^{1} \mathrm{H}$ NMR, ${ }^{13} \mathrm{C}$ NMR, FTIR, and elemental analysis.

\section{EXPERIMENTAL}

Required all reagents were obtained commercially. Solvents were purified and dried before being used. All melting points were taken in open capillaries and are uncorrected. Thin-layer chromatography (TLC, on aluminium plates precoated with silica gel, $60 \mathrm{~F}_{254}, 0.25 \mathrm{~mm}$ thickness) (Merck, Darmstadt, Germany) was used for monitoring the progress of all reactions, purity and 
homogeneity of the synthesized compounds; eluent-hexane:ethyl acetate: (3:7). UV radiation and/or iodine were used as the visualizing agents. Elemental analysis $(\% \mathrm{C}, \mathrm{H}, \mathrm{N})$ was carried out by Perkin-Elmer 2400 series-II elemental analyzer (Perkin-Elmer, USA) and all compounds are within $\pm 0.4 \%$ of theory specified. The IR spectra were recorded in $\mathrm{KBr}$ on a Perkin-Elmer Spectrum GX FT-IR Spectrophotometer (Perkin-Elmer, USA) and only the characteristic peaks are reported in $\mathrm{cm}^{-1}$. ${ }^{1} \mathrm{H}$ NMR and ${ }^{13} \mathrm{C}$ NMR spectra were recorded in DMSO- $d_{6}$ on a Bruker Avance $400 \mathrm{~F}(\mathrm{MHz})$ spectrometer (Bruker Scientific Corporation Ltd., Switzerland) using solvent peak as internal standard at $400 \mathrm{MHz}$ and $100 \mathrm{MHz}$ respectively. Chemical shifts are reported in parts per million (ppm). Mass spectra were scanned on a Shimadzu LCMS 2010 spectrometer (Shimadzu, Tokyo, Japan).

\subsection{Synthetic route for the synthesis of compound (1a)}

Dry ethanol $(10 \mathrm{ml})$, piperidine $(1 \mathrm{ml})$, Substituted benzene-1,2-diamine $(5 \mathrm{mmol})$, diethyl oxalate $(5 \mathrm{mmol})$ were charged in a $100-\mathrm{ml}$ round bottom flask with mechanical stirrer and condenser. The reaction mixture refluxed for $4 \mathrm{~h}$. After the completion of reaction (checked by TLC), the separated substituted quinoxaline-2,3(1H,4H)-dione was filtered and washed with ethanol and dried. Now thionyl chloride $(25 \mathrm{mmol})$ was added to a solution of substituted quinoxaline$2,3(1 H, 4 H)$-dione $(10 \mathrm{gm})$ in dry DCM $(50 \mathrm{~mL})$. Upon the addition of 1-2 drop of DMF a mixture was heated at reflux for $1 \mathrm{~h}$. After the completion of reaction a mixture was washed with water followed by saturated $\mathrm{NaHCO}_{3}$ solution. The organic phase was dried over anhydrous $\mathrm{Na}_{2} \mathrm{SO}_{4}$ and solvent was removed under reduced pressure to afford pure substituted 2,3-dichloroquinoxaline.

\subsection{Synthetic route for the synthesis of compound (3c)}

Dry potassium carbonate $(5 \mathrm{mmol})$ and dry DMF $(10 \mathrm{ml})$ were taken in $100 \mathrm{ml}$ RBF. Substituted 2,3-dichloroquinoxaline 1a $(5 \mathrm{mmol})$, 4-(thiophen-2-yl)thiazol-2-amine $\mathbf{2 b}(5 \mathrm{mmol})$, were added to this solution. The suspension then was heated at $100{ }^{0} \mathrm{C} \quad 4 \mathrm{~h}$. The reaction was monitored by TLC using Ethyl acetate: Hexane as a mobile phase. After completion of the reaction, reaction mixture was cooled. The separated solid was filtered, washed well with water and dried, and recrystallized from ethanol to afford analytically pure substituted 3-chloro- $N$-(4-(thiophen-2yl)thiazol-2-yl)quinoxalin-2-amine.

\subsection{General synthetic route for the synthesis of compounds 4(a-t)}

The mixture of substituted 3-chloro- $N$-(4-(thiophen-2-yl)thiazol-2-yl)quinoxalin-2-amine 3c ( $5 \mathrm{mmol}$ ) and hydrazine hydrate $(5 \mathrm{mmol})$ in ethanol was refluxed for $2 \mathrm{~h}$. on water bath. After completion of the reaction, solid product was appeared in the reaction. Cool the reaction mixture up to $\mathrm{rt}$ and filter the separated product washed with ethanol and dried at $\mathrm{rt}$ to furnished analytically pure substituted 3-hydrazinyl- $N$-(4-(thiophen-2-yl)thiazol-2-yl)quinoxalin-2-amine. Now substituted 3-hydrazinyl- $N$-(4-(thiophen-2-yl)thiazol-2-yl)quinoxalin-2-amine ( $5 \mathrm{mmol}$ ) and various aromatic aldehydes $(5 \mathrm{mmol})$ in ethanol was refluxed for $2 \mathrm{~h}$. on water bath. After completion of the reaction, solid product was appeared in the reaction. Cool the reaction mixture up to rt and filter the separated product washed with ethanol and dried at rt to furnished analytically pure 3-((E)-2benzylidenehydrazinyl)- $N$-(4-(thiophen-2-yl)thiazol-2-yl)quinoxalin-2-amine derivatives. Then to a solution of 3-((E)-2-benzylidenehydrazinyl)- $N$-(4-(thiophen-2-yl)thiazol-2-yl)quinoxalin-2-amine derivatives $(5 \mathrm{mmol})$ in MDC $(10 \mathrm{ml})$ was added Iodobenzene diacetate $(15 \mathrm{mmol})$ while stirring at rt. This solution was stirred for 45-55 minitues at rt. After the completion of reaction, monitored by the TLC, the mixture was washed with water followed by saturated $\mathrm{NaHCO}_{3}$ solution. The organic phase was dried over anhydrous $\mathrm{Na}_{2} \mathrm{SO}_{4}$ and solvent was removed under reduced pressure to afford solid product which recrystallized from chloroform to obtain pure compounds $\mathbf{4 a - 4 t}$. The physicochemical and spectral properties of all the newly synthesized compounds $\mathbf{4 a - 4 t}$. are presented below. 
<smiles>[R][R]1ccc2nc(Cl)c(Cl)nc2c1</smiles>

Where $\mathrm{R}=\mathrm{H}, \mathrm{CH}_{3}$

Scheme 1 Synthetic pathway for the synthesis of intermediate 1a<smiles>Clc1nc2c[R1]ccc2nc1Cl</smiles>

$1 \mathrm{a}$<smiles>Nc1nc(-c2cccs2)cs1</smiles>

2b<smiles>O=C(O)C=CC(=O)C(=O)O</smiles>

Where $\mathrm{R}=\mathrm{H}, \mathrm{CH}_{3}$<smiles>Clc1nc2cc[R]#cc2nc1Nc1nc(-c2cccs2)cs1</smiles>

3c

Scheme 2 Synthetic pathway for the synthesis of intermediate $3 \mathrm{c}$<smiles>Clc1nc2cc[R1]cc2nc1Nc1nc(-c2cccs2)cs1</smiles>

$3 c$<smiles></smiles>

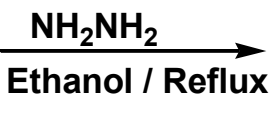

Ethanol / Reflux<smiles>NNc1nc2cc[R]#cc2nc1Nc1nc(-c2cccs2)cs1</smiles>

4a-4t

$$
\text { Where } \mathbf{R}=\mathbf{H}, \mathrm{CH}_{3}
$$

$\mathrm{Phl}(\mathrm{OAc})_{2}$ MDC / R.T.

$$
\text { Where } \mathrm{R}_{1}=\mathrm{H}, \mathrm{CH}_{3}, \mathrm{OCH}_{3}, \mathrm{~F}, \mathrm{Cl}, \mathrm{Br}, \mathrm{NO}_{2}, \mathrm{OH}
$$

Scheme 3 Synthetic pathway for the synthesis of thiophenyl thiazole depended triazolo quinoxaline derivatives $4 \mathrm{a}-4 \mathrm{t}$ 
Tabel 1 Synthesis of triazolo quinoxaline derivatives $4 \mathrm{a}-4 \mathrm{t}$

\begin{tabular}{|c|c|c|c|c|c|}
\hline Entry & $\mathbf{R}$ & $\mathbf{R}_{\mathbf{1}}$ & $\mathbf{R T}(\mathbf{m i n})$. & Yield \% & $\mathbf{m p}{ }^{\mathbf{0}} \mathbf{C}$ \\
\hline $4 \mathrm{a}$ & $\mathrm{H}$ & $\mathrm{H}$ & 46 & 89 & $130-132$ \\
\hline $4 \mathrm{~b}$ & $\mathrm{H}$ & $4-\mathrm{CH}_{3}$ & 45 & 90 & $142-144$ \\
\hline $4 \mathrm{c}$ & $\mathrm{H}$ & $4-\mathrm{OCH}_{3}$ & 47 & 88 & $124-126$ \\
\hline $4 \mathrm{~d}$ & $\mathrm{H}$ & $4-\mathrm{F}$ & 49 & 82 & $125-127$ \\
\hline $4 \mathrm{e}$ & $\mathrm{H}$ & $4-\mathrm{Cl}$ & 49 & 80 & $133-135$ \\
\hline $4 \mathrm{f}$ & $\mathrm{H}$ & $4-\mathrm{Br}$ & 48 & 81 & $138-142$ \\
\hline $4 \mathrm{~g}$ & $\mathrm{H}$ & $4-\mathrm{NO}_{2}$ & 47 & 84 & $133-135$ \\
\hline $4 \mathrm{~h}$ & $\mathrm{H}$ & $4-\mathrm{OH}$ & 48 & 82 & $150-152$ \\
\hline $4 \mathrm{i}$ & $\mathrm{H}$ & $3-\mathrm{NO}_{2}$ & 51 & 73 & $161-163$ \\
\hline $4 \mathrm{j}$ & $\mathrm{H}$ & $3-\mathrm{OH}$ & 51 & 71 & $157-159$ \\
\hline $4 \mathrm{k}$ & $\mathrm{CH}_{3}$ & $\mathrm{H}$ & 46 & 87 & $141-143$ \\
\hline $4 \mathrm{l}$ & $\mathrm{CH}$ & $4-\mathrm{CH}_{3}$ & 47 & 86 & $147-149$ \\
\hline $4 \mathrm{~m}$ & $\mathrm{CH}_{3}$ & $4-\mathrm{OCH}$ & 46 & 88 & $161-163$ \\
\hline $4 \mathrm{n}$ & $\mathrm{CH}_{3}$ & $4-\mathrm{F}$ & 50 & 82 & $135-137$ \\
\hline $4 \mathrm{o}$ & $\mathrm{CH}_{3}$ & $4-\mathrm{Cl}_{3}$ & 48 & 83 & $115-117$ \\
\hline $4 \mathrm{p}$ & $\mathrm{CH}_{3}$ & $4-\mathrm{Br}$ & 47 & 80 & $149-151$ \\
\hline $4 \mathrm{q}$ & $\mathrm{CH}_{3}$ & $4-\mathrm{NO}_{2}$ & 48 & 86 & $128-130$ \\
\hline $4 \mathrm{r}$ & $\mathrm{CH}_{3}$ & $4-\mathrm{OH}$ & 49 & 81 & $119-121$ \\
\hline $4 \mathrm{~s}$ & $\mathrm{CH}_{3}$ & $3-\mathrm{NO}_{2}$ & 52 & 74 & $167-169$ \\
\hline $4 \mathrm{t}$ & $\mathrm{CH}_{3}$ & $3-\mathrm{OH}$ & 54 & 70 & $143-145$ \\
\hline
\end{tabular}
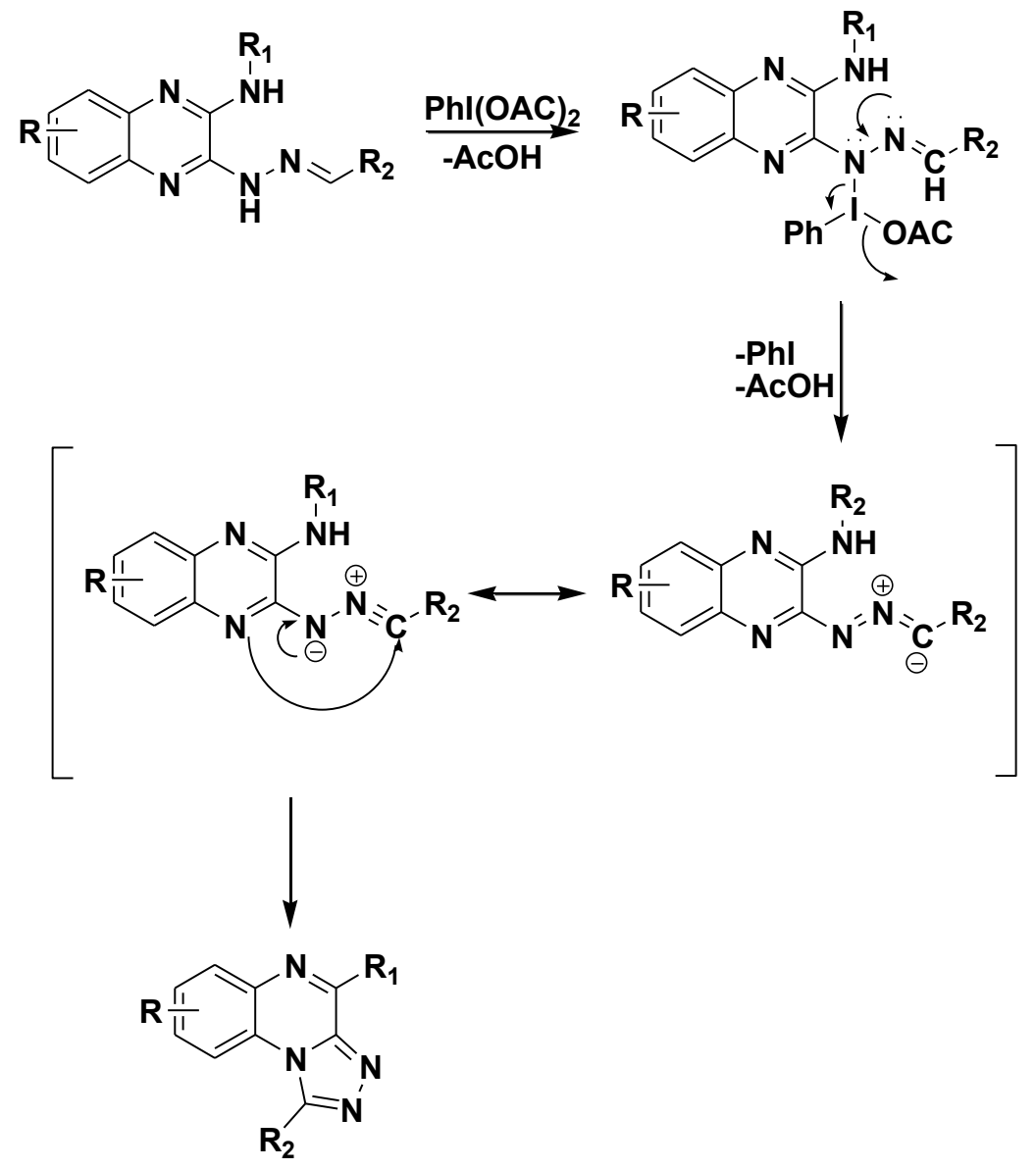

Where $\mathrm{R}=\mathrm{H}, \mathrm{CH}_{3}$

$R_{1}=$ Heterocyles

$\mathbf{R}_{\mathbf{2}}=$ Aryl

Scheme 4 Mechanism for the synthesis of thiophene thiazole depended triazolo quinoxaline derivatives $4 \mathrm{a}-4 \mathrm{t}$ 
The required 4-(thiophen-2-yl)thiazol-2-amine $\mathbf{2 b}$ was prepared by solid phase reaction according to literature procedure [19].

In this study, a series of triazolo quinoxaline derivatives $\mathbf{4 a - 4 t}$ has been synthesized by dehydrogenative cyclisation reaction of 3-((E)-2-benzylidenehydrazinyl)- $N$-(4-(thiophen-2yl)thiazol-2-yl)quinoxalin-2-amine derivatives using iodobenzene diacetate as catalyst (Scheme 3). In accordance with the mechanism suggested in (scheme-4), the first step of this process may involve electrophilic attack of iodobenzene diacetate on 3-((E)-2-benzylidenehydrazinyl)- $N$-(4(thiophen-2-yl)thiazol-2-yl)quinoxalin-2-amine derivatives to generates the nitrile imine. Then due to loss of iodobenzene and acetic acid, finally ring closure occurs through quinoxaline ring nitrogen and leads to the formation of product $\mathbf{4 a - 4 t}$.

The structures of all the new synthesized compounds were confirmed by ${ }^{1} \mathrm{H} N M R,{ }^{13} \mathrm{C}$ NMR, FTIR, elemental analysis, and molecular weight of some selected compounds confirmed by mass spectrometry. In ${ }^{1} \mathrm{H}$ NMR (DMSO-d6) spectrum of compound $\mathbf{4 c}$ exhibited singlet peak at $\mathrm{d}$ $10.30 \mathrm{ppm}$ for $-\mathrm{NH}-$ proton while multiplets around d 6.92-7.84 ppm for aromatic protons. Also exhibited singlet peak at d $3.70 \mathrm{ppm}$ for methoxy protons. In the ${ }^{13} \mathrm{C}$ NMR spectrum of compound 4c showed signals around d 109.22-142.10 ppm for aromatic carbons and d 57.94 for aromatic methoxy carben. The IR spectrum of compound $\mathbf{4 c}$ exhibited characteristic absorption band at 3265 $\mathrm{cm}^{-1}$ for cyclic $-\mathrm{NH}-$ and $3,038 \mathrm{~cm}^{-1}$ for aromatic $\mathrm{C}-\mathrm{H}$ stretching vibration. The mass spectra detected the expected molecular ion signals corresponding to respective molecular formula of synthesized compounds. Mass spectra of compound $\mathbf{4 c}$ gave molecular ion peak at $456.0(\mathrm{M}+1)$ corresponding to molecular formula $\mathrm{C}_{23} \mathrm{H}_{16} \mathrm{~N}_{6} \mathrm{OS}_{2}$. The obtained elemental analysis values are in good agreement with theoretical data. Similarly, all these compounds were characterized on the basis of spectral studies. All spectroscopic data have been given in spectral data.

\section{SPECTRAL DATA}

\section{1-phenyl- $N$-(4-(thiophen-2-yl)thiazol-2-yl)-[1,2,4]triazolo[4,3-a]quinoxalin-4-amine (4a)}

yellow solid, yield 89\%, m.p. 130-132 C, IR (KBr, v, cm ${ }^{-1}$ ): 3265 (N-H Str.), 3040 (Ar C-H Str.), ${ }^{1} \mathrm{H}$ NMR $\left(400 \mathrm{MHz}, \mathrm{DMSO}-d_{6}\right) \delta_{\mathrm{H}}(\mathrm{ppm}): \delta 6.713-7.698(\mathrm{~m}, 13 \mathrm{H}, \mathrm{Ar}-\mathrm{H}), 10.580(\mathrm{~s}, 1 \mathrm{H}, \mathrm{NH}) .{ }^{13} \mathrm{C}$ NMR $\left(100 \mathrm{MHz}, \mathrm{DMSO}-d_{6}\right) \delta_{\mathrm{C}}(\mathrm{ppm}): 110.23,111.10,112.03,113.26,115.20,116.90,118.30$, $119.90,122.00,123.60,125.66,127.33,128.69,129.35,132.01,133.65,134.88,136.04,137.28$, 138.33, 140.42, 143.00(Ar- $\underline{\mathrm{C}})$. $\mathrm{MS}\left(\mathrm{M}^{+}\right): 426.07$, Anal. Calcd. for $\mathrm{C}_{22} \mathrm{H}_{14} \mathrm{~N}_{6} \mathrm{~S}_{2}$ (426.52): C 61.95, $\mathrm{H}$ 3.31, N 19.70 Found: C 61.70, H 3.46, N 19.50\%.

\section{$N$-(4-(thiophen-2-yl)thiazol-2-yl)-1-p-tolyl-[1,2,4] triazolo[4,3-a]quinoxalin-4-amine (4b)}

yellow solid, yield 90\%, m.p. 142-144 C, IR (KBr, v, cm ${ }^{-1}$ ): 3214 (N-H Str.), 3026 (Ar C-H Str.), ${ }^{1} \mathrm{H}$ NMR $\left(400 \mathrm{MHz}, \mathrm{DMSO}-d_{6}\right) \delta_{\mathrm{H}}(\mathrm{ppm}): \delta 2.416\left(\mathrm{~s}, 3 \mathrm{H}, \mathrm{CH}_{3}\right), 6.800-7.945(\mathrm{~m}, 12 \mathrm{H}, \mathrm{Ar}-\mathrm{H})$, $10.600(\mathrm{~s}, 1 \mathrm{H}, \mathrm{NH}) .{ }^{13} \mathrm{C}$ NMR $\left(100 \mathrm{MHz}, \mathrm{DMSO}-d_{6}\right) \delta_{\mathrm{C}}(\mathrm{ppm}): 24.13\left(\mathrm{Ar}-\mathrm{CH}_{3}\right), 110.03,111.22$, 112.16, 114.66, 115.88, 116.99, 117.40, 119.10, 122.64, 123.14, 125.32, 126.56, 128.19, 130.32, 131.05, 132.66, 133.80, 136.04, 137.05, 138.13, 139.41, 142.06(Ar-C). MS( $\left.{ }^{+}\right)$: 440.09, Anal. Calcd. for $\mathrm{C}_{23} \mathrm{H}_{16} \mathrm{~N}_{6} \mathrm{~S}_{2}$ (440.54): C 62.71, H 3.66, N 19.08 Found: C 62.60, H 3.78, N 19.28\%.

\section{1-(4-methoxyphenyl)- $N$-(4-(thiophen-2-yl)thiazol-2-yl)-[1,2,4]triazolo[4,3-a]quinoxalin-4- amine (4c)}

yellow solid, yield 88\%, m.p. 124-126 C, IR (KBr, v, cm ${ }^{-1}$ ): 3265 (N-H Str.), 3038 (Ar C-H Str.), ${ }^{1} \mathrm{H}$ NMR (400 MHz, DMSO- $\left.d_{6}\right) \delta_{\mathrm{H}}(\mathrm{ppm}): \delta 3.700\left(\mathrm{~s}, 3 \mathrm{H}, \mathrm{OCH}_{3}\right), 6.920-7.845(\mathrm{~m}, 12 \mathrm{H}, \mathrm{Ar}-\mathrm{H})$, $10.300(\mathrm{~s}, 1 \mathrm{H}, \mathrm{NH}) .{ }^{13} \mathrm{C}$ NMR $\left(100 \mathrm{MHz}, \mathrm{DMSO}-d_{6}\right) \delta_{\mathrm{C}}(\mathrm{ppm}): 57.94\left(\mathrm{OCH}_{3}\right), 109.22,110.16$, $111.33,112.16,114.18,115.91,117.20,119.82$, 122.26, 123.33, 125.44, 127.17, 128.19, 129.45, 
132.64, 133.36, 134.63, 136.27, 137.22, 138.31, 139.41, 142.10(Ar-C). $\mathrm{MS}\left(\mathrm{M}^{+}\right)$: 456.08, Anal. Calcd. for $\mathrm{C}_{23} \mathrm{H}_{16} \mathrm{~N}_{6} \mathrm{OS}_{2}$ (456.54): C 60.51, H 3.53, N 18.41 Found: C 60.76, H 3.70, N 18.20\%

\section{1-(4-fluorophenyl)- $N$-(4-(thiophen-2-yl)thiazol-2-yl)-[1,2,4]triazolo[4,3-a]quinoxalin-4-amine} (4d)

yellow solid, yield 82\%, m.p. 125-127 C, IR (KBr, v, cm ${ }^{-1}$ ): 3268 (N-H Str.), 3042 (Ar C-H Str.), ${ }^{1} \mathrm{H}$ NMR $\left(400 \mathrm{MHz}, \mathrm{DMSO}-d_{6}\right) \delta_{\mathrm{H}}(\mathrm{ppm}): \delta$ 7.023-8.100 (m, 12H, Ar-H), $10.400(\mathrm{~s}, 1 \mathrm{H}, \mathrm{NH}) .{ }^{13} \mathrm{C}$ NMR $\left(100 \mathrm{MHz}, \mathrm{DMSO}-d_{6}\right) \delta_{\mathrm{C}}(\mathrm{ppm}): 106.23,107.40,109.12,110.24,113.16,114.98,116.80$, $118.00,120.13,121.16,123.31,124.80,126.34,128.18,129.12,131.18,132.15,133.00,135.18$, 137.01, 139.43, 141.12(Ar-ㅡ). $\mathrm{MS}\left(\mathrm{M}^{+}\right)$: 444.06, Anal. Calcd. for $\mathrm{C}_{22} \mathrm{H}_{13} \mathrm{FN}_{6} \mathrm{~S}_{2}$ (444.51): C 59.44, $\mathrm{H}$ 2.95, N 18.91 Found: C 59.55, H 3.20, N 18.50\%

\section{1-(4-chlorophenyl)- $N$-(4-(thiophen-2-yl)thiazol-2-yl)-[1,2,4] triazolo[4,3-a]quinoxalin-4-amine (4e)}

yellow solid, yield 80\%, m.p. 133-135 C, IR (KBr, v, cm ${ }^{-1}$ ): 3256 (N-H Str.), 3023 (Ar C-H Str.), ${ }^{1} \mathrm{H}$ NMR $\left(400 \mathrm{MHz}, \mathrm{DMSO}-d_{6}\right) \delta_{\mathrm{H}}(\mathrm{ppm}): \delta 7.012-8.100(\mathrm{~m}, 12 \mathrm{H}, \mathrm{Ar}-\mathrm{H}), 10.398(\mathrm{~s}, 1 \mathrm{H}, \mathrm{NH}) .{ }^{13} \mathrm{C}$ NMR $\left(100 \mathrm{MHz}, \mathrm{DMSO}-d_{6}\right) \delta_{\mathrm{C}}(\mathrm{ppm}): 106.88,107.65,109.55,110.21,113.55,115.90,116.84$, $118.26,120.65,121.33,123.24,124.55,126.12$, 128.13, 129.10, 130.10, 131.16, 133.12, 135.58, 137, 11, 139.46, 141.88(Ar-C). $\mathrm{MS}\left(\mathrm{M}^{+}\right)$: 460.03, Anal. Calcd. for $\mathrm{C}_{22} \mathrm{H}_{13} \mathrm{ClN}_{6} \mathrm{~S}_{2}$ (460.96): C 57.32, H 2.84, N 18.23 Found: C 57.20, H 3.02, N 18.35\%.

\section{1-(4-bromophenyl)- $N$-(4-(thiophen-2-yl)thiazol-2-yl)-[1,2,4]triazolo[4,3-a]quinoxalin-4-amine} (4f)

yellow solid, yield 81\%, m.p. 138-142 ${ }^{\circ} \mathrm{C}$, IR (KBr, v, $\mathrm{cm}^{-1}$ ): 3224 (N-H Str.), 3012 (Ar C-H Str.), ${ }^{1} \mathrm{H}$ NMR $\left(400 \mathrm{MHz}, \mathrm{DMSO}-d_{6}\right) \delta_{\mathrm{H}}(\mathrm{ppm}): \delta 6.978-7.800(\mathrm{~m}, 12 \mathrm{H}, \mathrm{Ar}-\mathrm{H}), 10.654(\mathrm{~s}, 1 \mathrm{H}, \mathrm{NH}) .{ }^{13} \mathrm{C}$ NMR $\left(100 \mathrm{MHz}, \mathrm{DMSO}-d_{6}\right) \delta_{\mathrm{C}}(\mathrm{ppm}): 109.34,111.02,113.24,114.12,115.36,116.24,117.30$, $118.54,119.90,121.20,123.00,124.56,126.47,128.03,130.13,132.45,133.85,136.30,138.18$, 140.16, 141.18, 142.89(Ar- $\underline{\text { C). }} \mathrm{MS}\left(\mathrm{M}^{+}\right)$: 503.98, Anal. Calcd. for $\mathrm{C}_{22} \mathrm{H}_{13} \mathrm{BrN}_{6} \mathrm{~S}_{2}$ (505.41): C 52.28, H 2.59, N 16.63 Found: C 52.36, H 2.72, N 16.80\%

\section{1-(4-nitrophenyl)- $N$-(4-(thiophen-2-yl)thiazol-2-yl)-[1,2,4]triazolo[4,3-a]quinoxalin-4-amine $(4 \mathrm{~g})$}

yellow solid, yield 84\%, m.p. 133-135 C, IR (KBr, v, $\mathrm{cm}^{-1}$ ): 3290 (N-H Str.), 3055 (Ar C-H Str.), ${ }^{1} \mathrm{H}$ NMR $\left(400 \mathrm{MHz}, \mathrm{DMSO}-d_{6}\right) \delta_{\mathrm{H}}(\mathrm{ppm}): \delta 7.145-8.124(\mathrm{~m}, 12 \mathrm{H}, \mathrm{Ar}-\mathrm{H}), 10.456(\mathrm{~s}, 1 \mathrm{H}, \mathrm{NH}) .{ }^{13} \mathrm{C}$ NMR $\left(100 \mathrm{MHz}, \mathrm{DMSO}-d_{6}\right) \delta_{\mathrm{C}}(\mathrm{ppm}): 110.31,111.25,113.36,115.02,115.96,116.33,117.80$, $118.94,119.20,122.10,123.50,124.46,125.40,127.06,129.10,131.15,132.86,134.40,137.19$, 141.16, 141.85, 141.80(Ar-ㄷ). $\mathrm{MS}\left(\mathrm{M}^{+}\right)$: 471.06, Anal. Calcd. for $\mathrm{C}_{22} \mathrm{H}_{13} \mathrm{~N}_{7} \mathrm{O}_{2} \mathrm{~S}_{2}$ (471.51): C 56.04, H 2.78, N 20.79 Found: C 56.10, H 2.85, N 20.88\%.

\section{4-(4-(4-(thiophen-2-yl)thiazol-2-ylamino)-[1,2,4]triazolo[4,3-a]quinoxalin-1-yl)phenol (4h)}

yellow solid, yield 82\%, m.p. 50-152 ${ }^{\circ}$, IR (KBr, v, $\mathrm{cm}^{-1}$ ): 3265 (N-H Str.), 3021 (Ar C-H Str.), ${ }^{1} \mathrm{H}$ NMR (400 MHz, DMSO- $\left.d_{6}\right) \delta_{\mathrm{H}}(\mathrm{ppm}): \delta 4.800(\mathrm{~s}, 1 \mathrm{H}, \mathrm{OH}), 7.005-8.100(\mathrm{~m}, 12 \mathrm{H}, \mathrm{Ar}-\mathrm{H}), 10.654$ $(\mathrm{s}, 1 \mathrm{H}, \mathrm{NH}) .{ }^{13} \mathrm{C}$ NMR $\left(100 \mathrm{MHz}, \mathrm{DMSO}-d_{6}\right) \delta_{\mathrm{C}}(\mathrm{ppm}): 106.35,108.36,110.23,112.10,114.76$, $116.34,117.50,118.61,119.14,121.23,123.40,124.50,126.34,128.01,130.22,132.41,133.80$, 134.20, 136.10, 138.19, 141.10, 141.98(Ar-ㅡ). $\mathrm{MS}\left(\mathrm{M}^{+}\right)$: 442.07, Anal. Calcd. for $\mathrm{C}_{22} \mathrm{H}_{14} \mathrm{~N}_{6} \mathrm{OS}_{2}$ (442.52): C 59.71, H 3.19, N 18.99 Found: C 59.60, H 3.30, N 19.20\%. 
1-(3-nitrophenyl)- $N$-(4-(thiophen-2-yl)thiazol-2-yl)-[1,2,4]triazolo[4,3-a]quinoxalin-4-amine (4i)

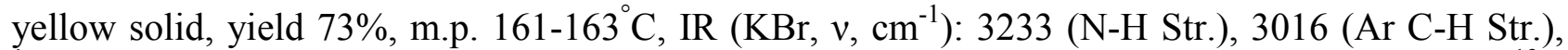
${ }^{1} \mathrm{H}$ NMR $\left(400 \mathrm{MHz}, \mathrm{DMSO}-d_{6}\right) \delta_{\mathrm{H}}(\mathrm{ppm}): \delta 7.140-8.042(\mathrm{~m}, 12 \mathrm{H}, \mathrm{Ar}-\mathrm{H}), 10.400 \mathrm{~S}(\mathrm{~s}, 1 \mathrm{H}, \mathrm{NH}) .{ }^{13} \mathrm{C}$ NMR $\left(100 \mathrm{MHz}, \mathrm{DMSO}-d_{6}\right) \delta_{\mathrm{C}}(\mathrm{ppm}): 113.18,115.20,116.25,117.24,119.80,121.34,122.00$, 124.26, 125.67, 127.18, 129.14, 130.12, 132.63, 134.16, 135.78, 137.22, 139.14, 141.50, 143.27, 144.00, 145.03, 146.28(Ar-C). $\mathrm{MS}\left(\mathrm{M}^{+}\right)$: 471.06, Anal. Calcd. for $\mathrm{C}_{22} \mathrm{H}_{13} \mathrm{~N}_{7} \mathrm{O}_{2} \mathrm{~S}_{2}$ (471.51): C 56.04, H 2.78, N 20.79 Found: C 56.32, H 2.90, N 20.92\%.

\section{3-(4-(4-(thiophen-2-yl)thiazol-2-ylamino)-[1,2,4] triazolo[4,3-a]quinoxalin-1-yl)phenol (4j)}

yellow solid, yield 71\%, m.p. $157-159^{\circ} \mathrm{C}$, IR ( $\mathrm{KBr}, v, \mathrm{~cm}^{-1}$ ): 3280 (N-H Str.), 3050 (Ar C-H Str.), ${ }^{1} \mathrm{H}$ NMR $\left(400 \mathrm{MHz}, \mathrm{DMSO}-d_{6}\right) \delta_{\mathrm{H}}(\mathrm{ppm}): \delta 4.980(\mathrm{~s}, 1 \mathrm{H}, \mathrm{OH}), 7.123-8.045(\mathrm{~m}, 12 \mathrm{H}, \mathrm{Ar}-\mathrm{H})$, $10.654(\mathrm{~s}, 1 \mathrm{H}, \mathrm{NH}) .{ }^{13} \mathrm{C}$ NMR $\left(100 \mathrm{MHz}, \mathrm{DMSO}-d_{6}\right) \delta_{\mathrm{C}}(\mathrm{ppm}): 112.31,114.21,116.20,117.33$, $119.81,121.30,122.22,123.16,125.65,127.10,129.11,130.06,132.55,134.32,135.18,136.12$, 138.24, 141.10, 142.22, 144.33, 145.58, 146.18(Ar- $\underline{\mathrm{C}})$. $\mathrm{MS}\left(\mathrm{M}^{+}\right)$: 442.07, Anal. Calcd. for $\mathrm{C}_{22} \mathrm{H}_{14} \mathrm{~N}_{6} \mathrm{OS}_{2}$ (442.52): C 59.71, H 3.19, N 18.99 Found: $\mathrm{C} 59.50, \mathrm{H} 3.33, \mathrm{~N} 18.80 \%$.

\section{8-methyl-1-phenyl- $N$-(4-(thiophen-2-yl)thiazol-2-yl)-[1,2,4] triazolo $[4,3-a]$ quinoxalin-4-amine (4k)}

yellow solid, yield 87\%, m.p. 141-143 ${ }^{\circ} \mathrm{C}$, IR (KBr, v, cm ${ }^{-1}$ ): 3266 (N-H Str.), 3014 (Ar C-H Str.), ${ }^{1} \mathrm{H}$ NMR $\left(400 \mathrm{MHz}, \mathrm{DMSO}-d_{6}\right) \delta_{\mathrm{H}}(\mathrm{ppm}): \delta 2.486\left(\mathrm{~s}, 3 \mathrm{H}, \mathrm{Ar}-\mathrm{CH}_{3}\right), 6.985-8.001(\mathrm{~m}, 12 \mathrm{H}, \mathrm{Ar}-\mathrm{H})$, $10.358(\mathrm{~s}, 1 \mathrm{H}, \mathrm{NH}) .{ }^{13} \mathrm{C}$ NMR $\left(100 \mathrm{MHz}, \mathrm{DMSO}-d_{6}\right) \delta_{\mathrm{C}}(\mathrm{ppm}): 26.10\left(\mathrm{Ar}-\mathrm{CH}_{3}\right), 112.30,114.32$, $115.60,116.36,118.30,119.00,120.54,121.00,123.06,125.66,126.90,127.88,130.00,131.65$, $132.55,134.03,136.98,138.00,139.01,142.30,143.00,145.01(\operatorname{Ar}-\underline{C}) . M S\left(M^{+}\right): 440.09$, Anal. Calcd. for $\mathrm{C}_{23} \mathrm{H}_{16} \mathrm{~N}_{6} \mathrm{~S}_{2}$ (440.54): C 62.71, H 3.66, N 19.08 Found: C 62.80, H 3.90, N 19.23\%.

\section{8-methyl- $N$-(4-(thiophen-2-yl)thiazol-2-yl)-1-p-tolyl-[1,2,4]triazolo[4,3-a]quinoxalin-4-amine (4I)}

yellow solid, yield 86\%, m.p. 147-149 ${ }^{\circ} \mathrm{C}$, IR (KBr, v, $\mathrm{cm}^{-1}$ ): 3288 (N-H Str.), 3023 (Ar C-H Str.), ${ }^{1} \mathrm{H}$ NMR (400 MHz, DMSO- $\left.d_{6}\right) \delta_{\mathrm{H}}(\mathrm{ppm}): \delta 2.420$ (s, 3H, Ar-CH $), 2.500$ (s, 3H, Ar-CH $\mathrm{CH}_{3}$ ), 6.900$7.845(\mathrm{~m}, 11 \mathrm{H}, \mathrm{Ar}-\mathrm{H}), 10.336(\mathrm{~s}, 1 \mathrm{H}, \mathrm{NH}) .{ }^{13} \mathrm{C}$ NMR $\left(100 \mathrm{MHz}, \mathrm{DMSO}-d_{6}\right) \delta_{\mathrm{C}}(\mathrm{ppm}): 25.30(\mathrm{Ar}-$ $\left.\mathrm{CH}_{3}\right), 27.14\left(\mathrm{Ar}-\mathrm{CH}_{3}\right), 111.23,112.55,113.62,115.30,116.10,118.12,120.55,121.22,123.18$, 125.54, 126.17, 127.32, 130.01, 131.33, 132.15, 134.18, 135.90, 138.10, 139.55, 142.20, 143.17, 145.21(Ar- $\underline{\mathrm{C}})$. $\mathrm{MS}\left(\mathrm{M}^{+}\right)$: 454.10, Anal. Calcd. for $\mathrm{C}_{24} \mathrm{H}_{18} \mathrm{~N}_{6} \mathrm{~S}_{2}$ (522.39): C 63.41, H 3.99, N 18.49 Found: C 63.52, H 4.09, N 18.85\%.

\section{1-(4-methoxyphenyl)-8-methyl- $N$-(4-(thiophen-2-yl)thiazol-2-yl)-[1,2,4]triazolo[4,3- a]quinoxalin-4-amine (4m)}

yellow solid, yield 88\%, m.p. 161-163 C, IR (KBr, v, cm ${ }^{-1}$ ): 3298 (N-H Str.), 3025 (Ar C-H Str.), ${ }^{1} \mathrm{H}$ NMR $\left(400 \mathrm{MHz}, \mathrm{DMSO}-d_{6}\right) \delta_{\mathrm{H}}(\mathrm{ppm}): \delta 2.451\left(\mathrm{~s}, 3 \mathrm{H}, \mathrm{Ar}-\mathrm{CH}_{3}\right), 4.002\left(\mathrm{~s}, 3 \mathrm{H}, \mathrm{OCH}_{3}\right), 7.001-$ $8.120(\mathrm{~m}, 11 \mathrm{H}, \mathrm{Ar}-\mathrm{H}), 10.500(\mathrm{~s}, 1 \mathrm{H}, \mathrm{NH}) .{ }^{13} \mathrm{C}$ NMR $\left(100 \mathrm{MHz}, \mathrm{DMSO}-d_{6}\right) \delta_{\mathrm{C}}(\mathrm{ppm}): 23.16(\mathrm{Ar}-$ $\left.\mathrm{CH}_{3}\right), 54.96\left(\mathrm{OCH}_{3}\right), 112.22,114.55,115.14,116.66,118.88,119.70,120.35,121.12,123.88$, $125.66,126.82,127.44,130.11,131.60,132.15,133.13,135.93,137.01,140.06,142.33,143.54$, 145.08(Ar-C). $\mathrm{MS}\left(\mathrm{M}^{+}\right)$: 470.10, Anal. Calcd. for $\mathrm{C}_{24} \mathrm{H}_{18} \mathrm{~N}_{6} \mathrm{OS}_{2}$ (470.57): C 61.26, H 3.86, N 17.86 Found: C 61.82, H 4.10, N 17.80\%. 
1-(4-fluorophenyl)-8-methyl- $N$-(4-(thiophen-2-yl)thiazol-2-yl)-[1,2,4]triazolo[4,3- $a]$ quinoxalin4-amine (4n)

yellow solid, yield 82\%, m.p. 135-137 $\mathrm{C}$, IR ( $\mathrm{KBr}, v, \mathrm{~cm}^{-1}$ ): 3214 (N-H Str.), 3038 (Ar C-H Str.), ${ }^{1} \mathrm{H}$ NMR $\left(400 \mathrm{MHz}, \mathrm{DMSO}-d_{6}\right) \delta_{\mathrm{H}}(\mathrm{ppm}): \delta 2.612\left(\mathrm{~s}, 3 \mathrm{H}, \mathrm{Ar}-\mathrm{CH}_{3}\right), 6.987-7.856(\mathrm{~m}, 11 \mathrm{H}, \mathrm{Ar}-\mathrm{H})$, $10.200(\mathrm{~s}, 1 \mathrm{H}, \mathrm{NH}) .{ }^{13} \mathrm{C}$ NMR $\left(100 \mathrm{MHz}, \mathrm{DMSO}-d_{6}\right) \delta_{\mathrm{C}}(\mathrm{ppm}): 27.16\left(\mathrm{Ar}-\mathrm{CH}_{3}\right), 111.00,112.69$, $114.03,116.48,117.80,119.20,121.37,122.36,124.30,126.39,127.89,129.60,131.33,133.12$, $135.14,137.18,139.15,140.12$, 141.20, 142.36, 144.08, 146.34(Ar- $\underline{\mathrm{C}})$. MS(M $\left.{ }^{+}\right)$: 458.08, Anal. Calcd. for $\mathrm{C}_{23} \mathrm{H}_{15} \mathrm{FN}_{6} \mathrm{~S}_{2}$ (458.53): C 60.25, H 3.30, N 18.33 Found: C 60.35, H 3.10, N 18.26\%.

1-(4-chlorophenyl)-8-methyl- $N$-(4-(thiophen-2-yl)thiazol-2-yl)-[1,2,4]triazolo[4,3-a]quinoxalin4-amine (40)

yellow solid, yield 83\%, m.p. 115-117 ${ }^{\circ} \mathrm{C}$, IR (KBr, v, $\mathrm{cm}^{-1}$ ): 3288 (N-H Str.), 3006 (Ar C-H Str.), ${ }^{1} \mathrm{H}$ NMR $\left(400 \mathrm{MHz}, \mathrm{DMSO}-d_{6}\right) \delta_{\mathrm{H}}(\mathrm{ppm}): \delta 2.456\left(\mathrm{~s}, 3 \mathrm{H}, \mathrm{Ar}-\mathrm{CH}_{3}\right), 6.900-7.890(\mathrm{~m}, 11 \mathrm{H}, \mathrm{Ar}-\mathrm{H})$, $10.356(\mathrm{~s}, 1 \mathrm{H}, \mathrm{NH}) .{ }^{13} \mathrm{C}$ NMR $\left(100 \mathrm{MHz}, \mathrm{DMSO}-d_{6}\right) \delta_{\mathrm{C}}(\mathrm{ppm}): 25.31\left(\mathrm{Ar}-\mathrm{CH}_{3}\right), 110.25,111.79$, $112.13,114.44,116.60,118.22,121.30,122.26,124.11,126.35,127.55,129.96,131.14,132.10$, 133.16, 136.20, 139.10, 140.22, 141.31, 142.30, 144.14, 146.20(Ar-C). $\mathrm{MS}\left(\mathrm{M}^{+}\right)$: 474.05, Anal. Calcd. for $\mathrm{C}_{23} \mathrm{H}_{15} \mathrm{ClN}_{6} \mathrm{~S}_{2}$ (474.99): C 58.16, H 3.18, N 17.69 Found: C 58.33, H 3.45, N 17.50\%.

\section{1-(4-bromophenyl)-8-methyl- $N$-(4-(thiophen-2-yl)thiazol-2-yl)-[1,2,4]triazolo[4,3- a]quinoxalin-4-amine (4p)}

yellow solid, yield 80\%, m.p. 149-151 ${ }^{\circ} \mathrm{C}$, IR (KBr, v, cm ${ }^{-1}$ ): 3241 (N-H Str.), 3033 (Ar C-H Str.), ${ }^{1} \mathrm{H}$ NMR $\left(400 \mathrm{MHz}, \mathrm{DMSO}-d_{6}\right) \delta_{\mathrm{H}}(\mathrm{ppm}): \delta 2.200\left(\mathrm{~s}, 3 \mathrm{H}, \mathrm{Ar}-\mathrm{CH}_{3}\right), 6.756-7.600(\mathrm{~m}, 11 \mathrm{H}, \mathrm{Ar}-\mathrm{H})$, $10.700(\mathrm{~s}, 1 \mathrm{H}, \mathrm{NH}) .{ }^{13} \mathrm{C}$ NMR $\left(100 \mathrm{MHz}, \mathrm{DMSO}-d_{6}\right) \delta_{\mathrm{C}}(\mathrm{ppm}): 24.26\left(\mathrm{Ar}-\mathrm{CH}_{3}\right), 107.64,109.13$, $111.02,112.36,114.15,116.18,117.20,119.65,121.30,122.08,124.15,126.33,127.69,128.77$, 130.14, 132.51, 133.87, 135.17, 136.45, 137.10, 139.40, 141.14(Ar-C). MS(M $\left.{ }^{+}\right)$: 518.00, Anal. Calcd. for $\mathrm{C}_{23} \mathrm{H}_{15} \mathrm{BrN}_{6} \mathrm{~S}_{2}$ (519.44): C 53.18, H 2.91, N 16.18 Found: C 53.23, H 3.00, N 16.03\%.

\section{8-methyl-1-(4-nitrophenyl)- $N$-(4-(thiophen-2-yl)thiazol-2-yl)-[1,2,4]triazolo[4,3-a]quinoxalin- 4-amine (4q)}

yellow solid, yield 86\%, m.p. 128-130 C, IR (KBr, v, $\mathrm{cm}^{-1}$ ): 3276 (N-H Str.), 3046 (Ar C-H Str.), ${ }^{1} \mathrm{H}$ NMR $\left(400 \mathrm{MHz}, \mathrm{DMSO}-d_{6}\right) \delta_{\mathrm{H}}(\mathrm{ppm}): \delta 2.600\left(\mathrm{~s}, 3 \mathrm{H}, \mathrm{Ar}-\mathrm{CH}_{3}\right), 6.600-7.020(\mathrm{~m}, 11 \mathrm{H}, \mathrm{Ar}-\mathrm{H})$, $10.345(\mathrm{~s}, 1 \mathrm{H}, \mathrm{NH}) .{ }^{13} \mathrm{C}$ NMR $\left(100 \mathrm{MHz}, \mathrm{DMSO}-d_{6}\right) \delta_{\mathrm{C}}(\mathrm{ppm}): 26.00\left(\mathrm{Ar}-\mathrm{CH}_{3}\right), 108.14,109.23$, $110.22,111.56,113.10,115.44,116.29,118.60,121.25,122.87,124.35,126.13,127.28,128.57$, $130.34,132.50,133.80,135.11,136.15,137.88,138.42,140.15(\operatorname{Ar}-\underline{C}) . M S\left(M^{+}\right): 485.07$, Anal. Calcd. for $\mathrm{C}_{23} \mathrm{H}_{15} \mathrm{~N}_{7} \mathrm{O}_{2} \mathrm{~S}_{2}$ (485.54): C 56.89, H 3.11, N 20.19 Found: C 56.60, H 3.20, N 20.28\%.

\section{4-(4-(4-(thiophen-2-yl)thiazol-2-ylamino)-8-methyl-[1,2,4]triazolo[4,3-a]quinoxalin-1- yl)phenol (4r)}

yellow solid, yield 81\%, m.p. 119-121 ${ }^{\circ} \mathrm{C}$, IR (KBr, v, $\mathrm{cm}^{-1}$ ): 3268 (N-H Str.), 3046 (Ar C-H Str.), ${ }^{1} \mathrm{H}$ NMR $\left(400 \mathrm{MHz}, \mathrm{DMSO}-d_{6}\right) \delta_{\mathrm{H}}(\mathrm{ppm}): \delta 2.563\left(\mathrm{~s}, 3 \mathrm{H}, \mathrm{Ar}-\mathrm{CH}_{3}\right), 4.500(\mathrm{~s}, 1 \mathrm{H}, \mathrm{OH}), 6.980-7.800$ $(\mathrm{m}, 11 \mathrm{H}, \mathrm{Ar}-\mathrm{H}), 10.212(\mathrm{~s}, 1 \mathrm{H}, \mathrm{NH}) .{ }^{13} \mathrm{C}$ NMR $\left(100 \mathrm{MHz}, \mathrm{DMSO}-d_{6}\right) \delta_{\mathrm{C}}(\mathrm{ppm}): 25.30\left(\mathrm{Ar}-\mathrm{CH}_{3}\right)$, $106.60,108.10,109.65,111.36,113.18,115.20,117.36,119.66,121.33,122.51,124.25,126.40$, $127.61,128.19,130.54,131.50,133.80,134.10,136.20,137.88,138.30,140.19($ Ar-C $\left.) . \mathrm{MS}^{+} \mathrm{M}^{+}\right)$: 456.08, Anal. Calcd. for $\mathrm{C}_{23} \mathrm{H}_{16} \mathrm{~N}_{6} \mathrm{OS}_{2}$ (456.54): C 60.51, H 3.53, N 18.41 Found: $\mathrm{C} 60.72, \mathrm{H} 3.74$, N $18.20 \%$. 
8-methyl-1-(3-nitrophenyl)- $N$-(4-(thiophen-2-yl)thiazol-2-yl)-[1,2,4]triazolo[4,3-a]quinoxalin4-amine (4s)

yellow solid, yield 74\%, m.p. IR (KBr, v, $\mathrm{cm}^{-1}$ ): 3250 (N-H Str.), 3040 (Ar C-H Str.), ${ }^{1} \mathrm{H}$ NMR (400 MHz, DMSO- $\left.d_{6}\right) \delta_{\mathrm{H}}(\mathrm{ppm}): \delta 2.400\left(\mathrm{~s}, 3 \mathrm{H}, \mathrm{Ar}-\mathrm{CH}_{3}\right), 7.002-8.100(\mathrm{~m}, 11 \mathrm{H}, \mathrm{Ar}-\mathrm{H}), 10.220(\mathrm{~s}, 1 \mathrm{H}$, $\mathrm{NH}) .{ }^{13} \mathrm{C}$ NMR $\left(100 \mathrm{MHz}, \mathrm{DMSO}-d_{6}\right) \delta_{\mathrm{C}}(\mathrm{ppm}): 26.00\left(\mathrm{Ar}-\mathrm{CH}_{3}\right), 110.34,112.26,113.68,115.02$, 117.18, 119.30, 121.45, 123.36, 125.41, 126.37, 128.12, 129.40, 131.25, 132.16, 133.88, 135.40, 137.20, 138.16, 140.12, 141.33, 143.00, 145.11(Ar- $\underline{\mathrm{C}}) . \mathrm{MS}\left(\mathrm{M}^{+}\right)$: 485.07 Anal. Calcd. for $\mathrm{C}_{23} \mathrm{H}_{15} \mathrm{~N}_{7} \mathrm{O}_{2} \mathrm{~S}_{2}$ (485.54): C 56.89, H 3.11, N 20.19 Found: C 56.70, H .20, N 20.30\%.

\section{3-(4-(4-(thiophen-2-yl)thiazol-2-ylamino)-8-methyl-[1,2,4]triazolo[4,3-a]quinoxalin-1- yl)phenol (4t)}

yellow solid, yield 70\%, m.p. 143-145 C, IR (KBr, v, $\mathrm{cm}^{-1}$ ): 3288 (N-H Str.), 3047 (Ar C-H Str.), ${ }^{1} \mathrm{H}$ NMR $\left(400 \mathrm{MHz}, \mathrm{DMSO}-d_{6}\right) \delta_{\mathrm{H}}(\mathrm{ppm}): \delta 2.560\left(\mathrm{~s}, 3 \mathrm{H}, \mathrm{Ar}-\mathrm{CH}_{3}\right), 4.400(\mathrm{~s}, 1 \mathrm{H}, \mathrm{OH}), 7.010-8.005$ $(\mathrm{m}, 11 \mathrm{H}, \mathrm{Ar}-\mathrm{H}), 10.546(\mathrm{~s}, 1 \mathrm{H}, \mathrm{NH}) .{ }^{13} \mathrm{C}$ NMR $\left(100 \mathrm{MHz}, \mathrm{DMSO}-d_{6}\right) \delta_{\mathrm{C}}(\mathrm{ppm}): 23.98\left(\mathrm{Ar}-\mathrm{CH}_{3}\right)$, $110.55,111.20,112.24,114.05,116.10,117.35,119.40,121.30,123.51,125.36,127.15,129.20$, $131.00,132.34,133.85,135.46,137.21,138.22,139.03,141.53,144.00,145.99($ Ar-C $) . \mathrm{MS}^{+}\left(\mathrm{M}^{+}\right)$: 456.08, Anal. Calcd. for $\mathrm{C}_{23} \mathrm{H}_{16} \mathrm{~N}_{6} \mathrm{OS}_{2}$ (456.54): C 60.51, H 3.53, N 18.41 Found: $\mathrm{C}$ 60.62, H 3.60, $\mathrm{N} 18.52 \%$.

\section{CONCLUSION}

In summary, novel series of triazolo quinoxalines having thiophene and thiazole moiety synthesized by dehydrogenative cylisation reaction through iodobenzene diacetate. The synthetic method produced a single scaffold with triazole, quinoxaline ,thophene and thiazole hetrocyclic systems.

\section{Acknowledgments}

The authors are thankful to the Head, Department of Chemistry, Saurashtra University, Rajkot for providing ${ }^{1} \mathrm{H}$ NMR , ${ }^{13} \mathrm{C}$ NMR, mass and FT-IR spectroscopy and research facilities.

\section{References}

[1] Pozharskii A., Soldatenkov A. T., Katritzky A. R., Heterocycles in life and society, Chichester, UK: John Wiley and Sons. (1997).

[2] Wiseloge F. W., et al., Survey of antimalarial drugs, 1941-1945. In: Armarego WLF, editor. Advances in hetero cyclic chemistry. 1 (1963) 304.

[3] Burguete A., Pontiki A., Litina D. H., Villar H., Vicente E., Solano B., Bioorganic and Medicinal Chemistry Letters. 17 (2007) 6439.

[4] Lindsley C. W., Zhao Z., Leister W. H., Robinson R. G., Barnett S. F., Bioorganic and Medicinal Chemistry Letters. 15 (2005) 761.

[5] Harmenberg J., Akesson-Johansson A., Graslund A., Malmfors T., Antiviral Research. 15 (1991) 193.

[6] Seitz L. E., Suling W. J., Journal of Medicinal Chemistry.45 (2002) 5604.

[7] More S. V., Sastry M. N. V., Wang C. C., Tetrahedron Letters. 46 (2005) 6345.

[8] Gris J., Glisoni R., Fabian L., Fernandez B., Tetrahedron Letters. 49 (2008) 1053.

[9] Sakata G., Makino K., Heterocycles. 27 (1988) 2481.

[10] Morkos A., Serry H., Med. Chem. Res. 21 (2012) 2368. 
[11] Catarzi D., Cecchi L., J. Med. Chem. 37 (1994) 2846.

[12] Cecchi L., Filacchioni G., Galli A., J. Med. Chem. 23 (2000) 3824.

[13] Galli A., Costagli C., Carla V., J. Med. Chem. 44 (2001) 3157.

[14] Mohamed A., Ghiaty A., El-Morsy A., ISRN Organic Chemistry. 2013 (2013) 7.

[15] Katritzky A., Tian-Bao H., Denisko V., J. Org. Chem. 67 (2002) 3118.

[16] Krishnan V. H. S., Chowdary K. S., Indian Journal of Chemistry. 398 (2000) 329.

[17] Eakta., Aggarwal R., Indian Journal of Chemistry. 52B (2013) 273.

[18] Praveen Kumar Darsi S. S., Andra N., Dubey P., Bhoomireddy R., International Journal of Chemical Engineering and Applications. 3 (2012) 6.

[19] Dodson R. M., King L. C., J. Am. Chem. Soc. 67 (1945) 2242.

[20] Khunt H., Pipaliya P., Ghelani S., Naliapara Y. T., International Letters of Chemistry, Physics and Astronomy 24 (2014) 134.

[21] Patel A. S., Khunt H., Babariya J., Ghelani S., Naliapara Y. T., International Letters of Chemistry, Physics and Astronomy 30 (2014) 106.

[22] Gami P., Vilapara K., Khunt H., Babariya J., Naliapara Y. T., International Letters of Chemistry, Physics and Astronomy 30 (2014) 127.

[23] Khunt H., Babariya J., Dedakiya C., Naliapara Y. T., International Letters of Chemistry, Physics and Astronomy 30 (2014) 185.

[24] Khunt H., Babariya J., Naliapara Y. T., International Letters of Chemistry, Physics and Astronomy 31 (2014) 20.

[25] Prajapati D., Vilapara K., Naliapara Y. T., International Letters of Chemistry, Physics and Astronomy 33 (2014) 12. 